\title{
Tipos y formas de ambientes de dormir de majás (Cuniculus paca) en la cuenca alta del río Itaya
}

\author{
Types and forms of sleeping dens of pacas (Cuniculus paca) in the upper \\ Itaya river basin
}

\author{
Rolando Aquino ${ }^{1}$, Gendrick Meléndez ${ }^{2}$, Etersit Pezo² y Deyber Gil²
}

1 Instituto de Ciencias Biológicas Antonio Raimondi, Facultad de Ciencias Biológicas, Universidad Nacional Mayor de San Marcos. P. O. Box 575 Iquitos, Perú Telefax 65265510. Email Rolando Aquino: uinoy2005@yahoo.es

2 Facultad de Ciencias Biológicas, Universidad Nacional de la Amazonía Peruana, Iquitos, Perú.
Presentado: $\quad$ 07/01/2012

$27 / 08 / 2012$

Publicado online: 01/10/2012

\section{Resumen}

Este reporte trata sobre ambientes de dormir de majás (Cuniculus paca, Linnaeus, 1766). El estudio fue llevado a cabo entre agosto del 2006 y abril del 2007 y está basado en observaciones detalladas. Los resultados indican que este roedor utiliza como ambientes de dormir los huecos en tierra o en troncos de árboles caídos. Cada uno de estos ambientes contaba con dos o más orificios y una cavidad interna para el "sueño diurno". Generalmente uno de los orificios era de uso habitual y el resto para la fuga circunstancial. De acuerdo a su forma, número de orificios y ubicación en el piso del bosque, los ambientes de dormir fueron clasificados hasta en cuatro tipos; de ellos, el tipo A fue el más común. Los resultados también indican mayor concentración de ambientes de dormir en bosque de galería de inundación temporal.

Palabras clave: Cuniculus paca, ambientes de dormir, caracterización, tipos y formas, concentración por hábitats.

\section{Abstract}

This report deals with paca sleeping dens (Cuniculus paca Linnaeus, 1766). The study was conducted between August 2006 and April 2007 and is based on detailed observations. Results indicate that this rodent uses holes in the ground or in fallen trees as sleeping dens. Each of these sleeping dens had two or more orifices and an internal cavity for the "diurnal sleep". Typically, one orifice was for common use and the rest for the circumstantial escape. According to their form, their localization in the ground, and the number and location of orifices, sleeping dens were classified into four types, of which type A was the most common. The results also indicate a greater concentration of sleeping dens in temporary flooded gallery forest.

Keywords: Cuniculus paca, sleeping dens, characterization, types and forms, habitat concentration.

\section{Introducción}

Entre los animales silvestres que habitan en los bosques amazónicos se encuentra el majás (Cuniculus paca, Rodentia: Cuniculidae) cuya carne tiene una alta demanda en los mercados por su exquisito sabor, por lo que la caza de este roedor se incrementó en estos últimos años, convirtiéndose así en una fuente importante de ingreso económico, en particular para aquellos pobladores que habitan en determinadas cuencas de la Amazonía (Bodmer et al. 1997). Sin embargo, esta extracción desmedida estaría generando sobre caza en algunas cuencas de la Amazonía peruana, por lo que es importante conocer con más detalle ciertos aspectos ecológicos como es el caso de los ambientes donde realizan el descanso o "sueńo diurno", que podría contribuir en la formulación de estrategias para un mejor uso de este importante recurso natural.

En cuanto a este roedor, la mayoría de los estudios realizados en la Amazonía peruana están referidos a la biomasa extraída (Bodmer et al. 1997; Aquino et al. 2001), comercialización en mercados (Bendayán 1991; Bendayán \& Bardales 2004)y sustentabilidad de la caza (Aquino et al., 2009); pero es muy escasa en lo que se refiere a las características de los ambientes de dormir que son mencionados de manera general por Mondolfi (1972), Boher (1981), Yockteng (1982), entre otros; así como a su distribución con respecto a los ríos, quebradas, caños y lagunas (cochas), que con excepción de Aquino et al. (2009) son comentados a grandes rasgos por Kroll y Giesecke (1996), Voss y Emmons (1996), Oliveira y Bonvicino (2006), entre otros. La escasa información disponible sobre los ambientes de dormir nos motivó a la conducción de este estudio, el cual estuvo orientado a la caracterización en tipos y formas. El estudio fue conducido de agosto 2006 hasta abril del 2007, cuyos resultados se presentan en este reporte.

\section{Material y métodos}

Área de estudio.- El estudio se llevó acabo en la cuenca alta del río Alto Itaya, localizada al sureste de la ciudad de Iquitos (Fig.1). En el área fueron diferenciados hasta tres tipos de hábitats, el bosque de terraza baja incluyendo el ribereño o de galería, bosque de terraza alta y bosque de colina baja de moderado a fuertemente disectado. Estos bosques se caracterizaron por poseer abundantes cuerpos acuáticos conformados por quebradas y caños, donde las diversas comunidades vegetales constituyeron hábitats y refugios para muchos componentes de la fauna silvestre, entre ellos el majás (C. paca). La composición florística varió entre uno y otro tipo de bosque, donde predominaron árboles de fuste casi rectos y entre 10 a $25 \mathrm{~m}$ de altura, pero también hubieron algunos emergentes superior a los $30 \mathrm{~m}$ como el machimango (Eschweilera sp., Gustavia sp.), pashaco (Parkia sp.), quinilla (Chrysophyllum sp., Pouteria sp.), entre otros.

Selección de unidades de muestreo para búsqueda de ambientes de dormir.- Para este propósito fue tomado en cuenta 


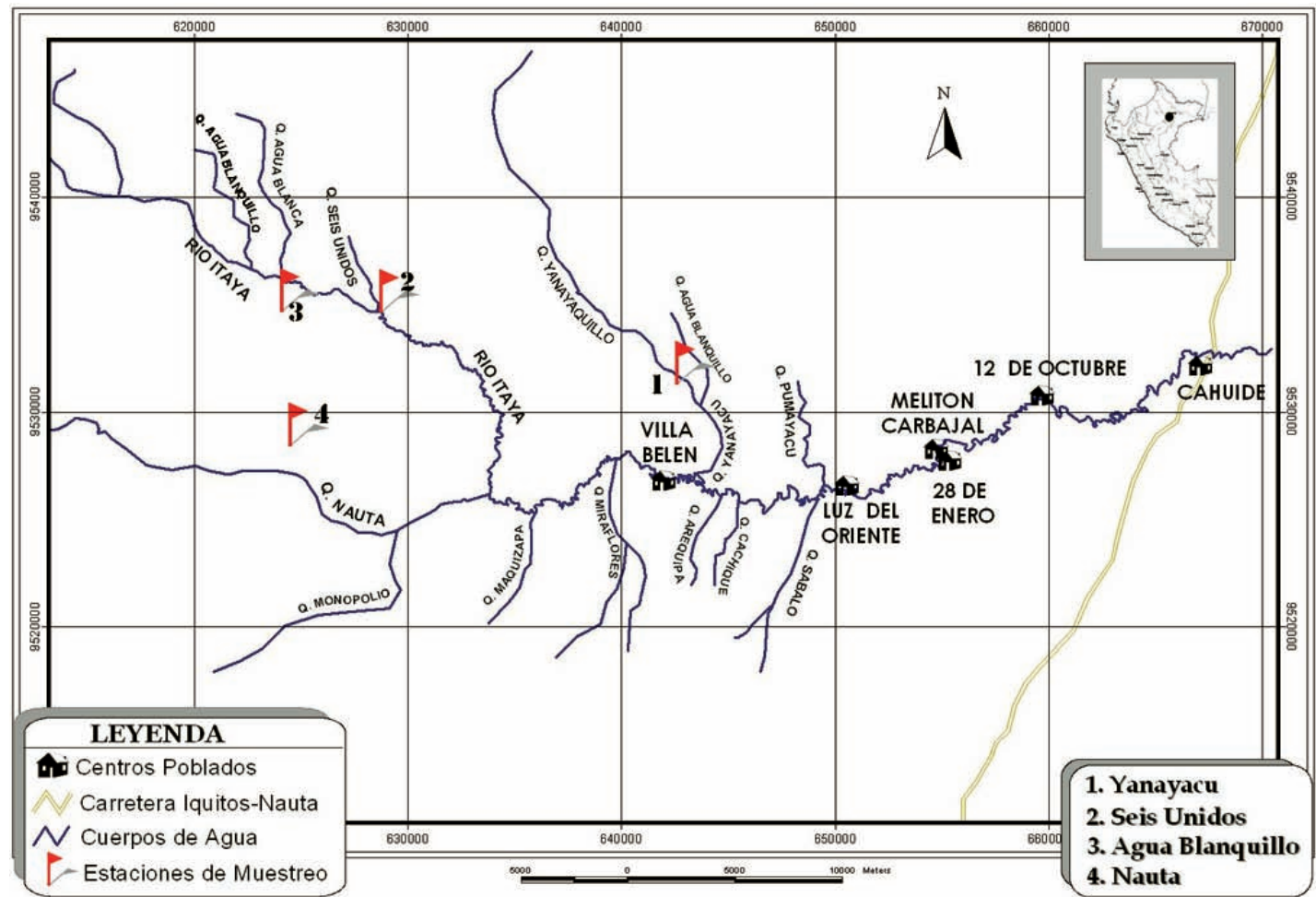

Figura 1. Mapa de la cuenca del río Alto Itaya mostrando las zonas de muestreo: 1) Quebrada Yanayacu, 2) Quebrada Seis Unidos, 3) Quebrada Agua Blanquillo y 4) Quebrada Nauta.

las estaciones de muestreo establecidos en estudios anteriores (Terrones 2006; Navarro \& Terrones 2006; Aquino et al. 2007), lo que facilitó la definición de las unidades de muestreo para el cual se tuvo en cuenta los siguientes: accesibilidad, en particular durante la época de vaciante (mayo - setiembre) y antecedentes sobre observación directa y/o indirecta de este roedor en estudios anterioes.

Considerando los criterios arriba mencionados, la búsqueda de ambientes de dormir se hizo en cuatro zonas de muestreo: quebrada Yanayacu (bosque de terraza alta), quebrada Seis Unidos (bosque de terraza baja que incluye el bosque de galería), quebrada Agua Blanquillo (bosque de colina baja fuertemente disectado) y quebrada Nauta (bosque de colina baja moderadamente disactado). La búsqueda y caracterización de ambientes de dormir comprendió desde agosto del 2006 hasta abril del 2007 de forma intercalada y no mensual, de modo que se hicieron seis viajes de 20 días útiles de trabajo de campo por cada tipo de bosque o zona de muestreo. En las unidades de muestreo previamente definidas se abrieron seis transectos de 2 $\mathrm{km}$ de longitud aledaños al río y quebradas y hacia el interior del bosque. Por cada uno de estos transectos se procedió a la búsqueda de ambientes de dormir con el apoyo de dos guías de campo que fueron cazadores de las comunidades aledañas al área de estudio. La búsqueda se hizo en una amplitud aproximada de $30 \mathrm{~m}$; es decir, $15 \mathrm{~m}$ por cada lado del transecto. Para este propósito, cada uno de los transectos fue recorrido de 08:00 a 14:00 h. Cada vez que se encontró un ambiente de dormir fue necesario verificar el uso actual ya sea con la presencia del animal en la cavidad interna o la fuga, para el cual en ocasiones recurrimos al hostigamiento mediante la incrustación de una rama delgada por los orificios. El uso del ambiente también fue verificado por la presencia de huellas recientes en la entrada del orificio de uso habitual.
Registro de ambientes de dormir.- Una vez confirmada el uso del ambiente se procedió a tomar datos referidos al origen; es decir, si fue consecuencia del cavado por armadillo (Dasypus spp.), erosión por el agua, descomposición del interior de un tronco del árbol caído, adopción de diversas formas de las raíces zancos de los árboles vivos y los montículos de tierra. Luego se procedió a la caracterización, por lo que fue necesario hacer cortes para inspeccionar la cavidad interna y la ubicación de los orificios de fuga, acción que demandó el empleo de 2 a 3 horas por cada ambiente de dormir. Los datos fueron anotados en una ficha de campo y estuvieron referidos al número de orificios de uso habitual (diferenciado del resto por el desgaste y trajín), número de orificios para la fuga circunstancial(diferenciada del anterior por encontrarse tapado con hojarascas), distancia de los orificios de fuga con respecto al cuerpo de agua, distancia entre orificios, diámetro de los orificios, ubicación del dormidero, presencia de restos orgánicos (heces, restos de frutos y semillas) y de otros animales con los que podrían cohabitar.

Caracterización de los ambientes de dormir.- Los ambientes de dormir fueron caracterizados in situ, señalando vías de entrada, de fuga, tipo y forma, longitud de las vías de acceso y de fuga, profundidad y presencia de hojarascas. Los datos obtenidos a través del estudio se analizaron utilizando las técnicas y métodos de la estadística descriptiva como la media aritmética, sumatoria, cuadros de distribución, entre otros. Para tal efecto se contó con el Programa Estadístico BioEstat versión 2,0.

\section{Resultados}

Descripción de un ambiente de dormir.- Como resultado de las actividades de campo fueron registrados 74 ambientes de dormir, cada uno de ellos compuesto por orificios, túneles y una cavidad interna donde se encontraba el sitio de dormir o dormidero. 
Orificios: ubicados en la parte anterior, laterales y posterior del ambiente de dormir. Estos orificios cumplieron dos funciones, algunos para la entrada hacia la cavidad interna y salida y otros para la fuga circunstancial. Los orificios de entrada o de acceso hacia la cavidad interna siempre estuvieron ubicados en la parte anterior del ambiente de dormir y por lo general fue una abertura sin obstáculos; es decir, libre de ramas y hojarascas. Estos orificios generalmente tenían la forma circular, con diámetro que fluctuaron entre 25 a $50 \mathrm{~cm}$, de aspecto limpio y libre de hojarascas; fue en esta parte del ambiente donde casi siempre se observaron huellas recientes de este roedor, confirmando así el uso actual cuando no fue sorprendido dentro de la cavidad interna o en plena fuga. Contrario al anterior, los orificios de fuga estuvieron ubicados en la parte posterior o laterales y hubo hasta cuatro en un ambiente de dormir. Estos orificios de forma circular y con diámetros que fluctuaron entre 8 a $10 \mathrm{~cm}$ casi siempre se encontraron tapados con hojarascas, mientras que en algunos casos pasaron desapercibidos debido a la lejanía de éstos con respecto al orificio de uso habitual.

Cavidad interna: conformado por el espacio vacío, generalmente de forma circular y con un diámetro mayor que el orificio de acceso donde se encontraba el dormidero formado por un colchón de hojarascas. Esta cavidad de aspecto limpio y seco, algunas veces estaba conectado con los orificios a través de túneles y en ellos ocasionalmente se encontraron arácnidos, hormigas y pequeńos reptiles y anfibios.

Principales tipos de ambientes de dormir.- Los 74 ambientes registrados fueron clasificados en cuatro principales tipos. El criterio para la tipificación de estos ambientes estuvo basado en las descripciones de uso de dormideros del género Aotus establecidos por Aquino y Encarnación (1986) y Puertas (1987). De acuerdo con este criterio, los ambientes fueron agrupados en los tipos A, B, C y D y sus respectivas nueve formas. Los diferentes tipos fueron registrados en montículos de tierra, entrecruzamiento de raíces zancos de árboles caídos y/o en pié y troncos secos de árboles caídos de gran porte incluyendo palmeras.

\section{Tipo A}

Conformado por huecos en montículos de tierra y por debajo del suelo. En este caso el roedor ingresaba a la cavidad interna por uno o dos orificios de forma casi circular o ligeramente ovalada y siempre estuvieron descubiertos, en tanto que los orificios de fuga ubicados en la parte posterior del ambiente estaban tapados con hojarascas, dando las condiciones óptimas para el resguardo y proporcionando seguridad ante los depredadores. En este tipo se diferenciaron hasta cuatro formas particulares:

Forma Aa: a manera de "Y" por la disposición de los túneles que conectaban con la cavidad interna donde se encontraba el dormidero; el orificio de uso habitual estuvo libre de obstáculos y los orificios de fuga que variaron desde uno a tres estaban tapados con hojarascas (Fig. 2). Por lo general dentro de la cavidad interna había un dormidero que sirvió al roedor para conciliar el "sueño diurno", el mismo que estaba muy cerca al orifico de uso habitual $(0,80 \mathrm{~m})$ e interconectada con los orificios de fuga por medio de túneles. El dormidero cuyo diámetro varió entre 0,20 a $0,80 \mathrm{~m}$ siempre estaba compuesto por un colchón de hojarascas. La distancia promedio entre los orificios de fuga fue de $1,84 \mathrm{~m}$, lo que indica el comportamiento eludible que posee este animal

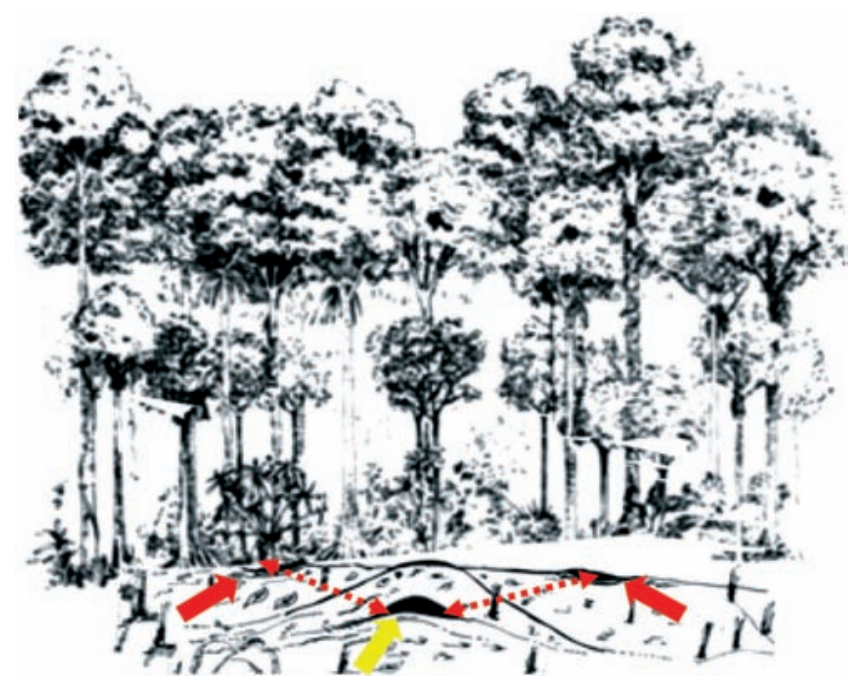

Figura 2. Gráfico de un ambiente de dormir de la forma Aa. Las flechas amarilla y roja indican los orificios de uso habitual y de fuga, respectivamente y las líneas roja punteadas los túneles que conectan con los orificios.(Dibujo de Meléndez G, 2006-2007)

ante sus depredadores. Por otro lado, la ubicación y forma del ambiente de dormir evitaba el escurrimiento directo del agua hacia la cavidad interna. Esta forma de ambiente de dormir fue observada con frecuencia en bosques de terraza alta.

Forma Ab: de aspecto laberíntico, cuyos orificios de uso habitual variaron de uno a cuatro, todos libres de hojarascas. En esta forma, los dormideros cuyo diámetro varió entre 0,20 y $0,30 \mathrm{~m}$ estaban interconectados a un complejo sistema de túneles y éstos a los orificios de fuga que se encontraban tapados con hojarascas (Fig. 3). En este caso la presencia de más de un orificio de uso habitual conectado a túneles permitía al roedor contar con más de un dormidero para el "sueńo diurno". Cabe mencionar que esta forma de ambiente de dormir con más de dos dormideros no fue común, siendo registrado en bosque de terraza alta.

Forma Ac: en esta forma el orificio de uso habitual algunas veces estuvo tapado con hojarascas, en tanto que la cavidad interna donde se encontraba el dormidero estaba conectado

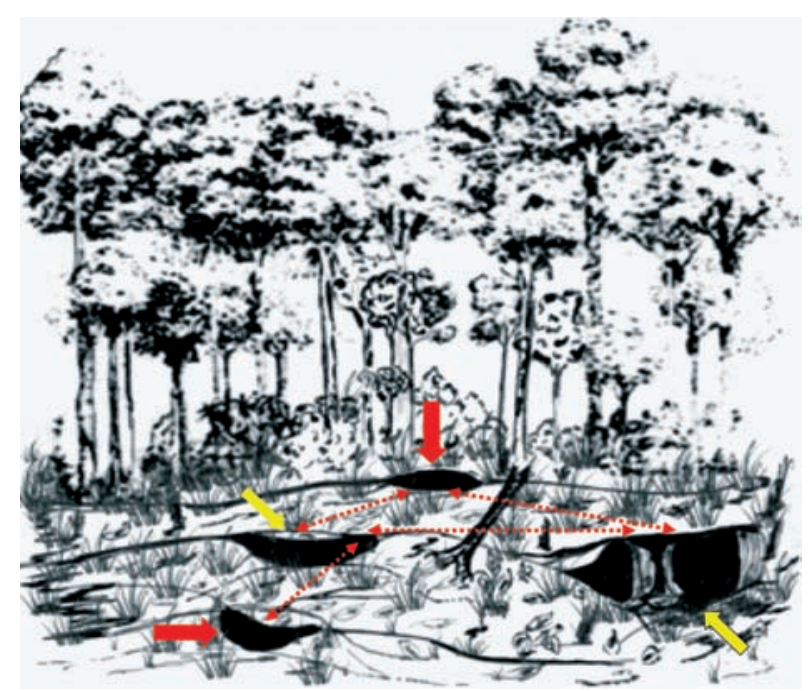

Figura 3. Gráfico de un ambiente de dormir de la forma Ab. Las flechas amarilla y roja indican los orificios de uso habitual y de fuga, respectivamente y las líneas roja punteadas la dirección de los túneles que conectan con los orificios. (Dibujo de Meléndez G, 2006-2007) 


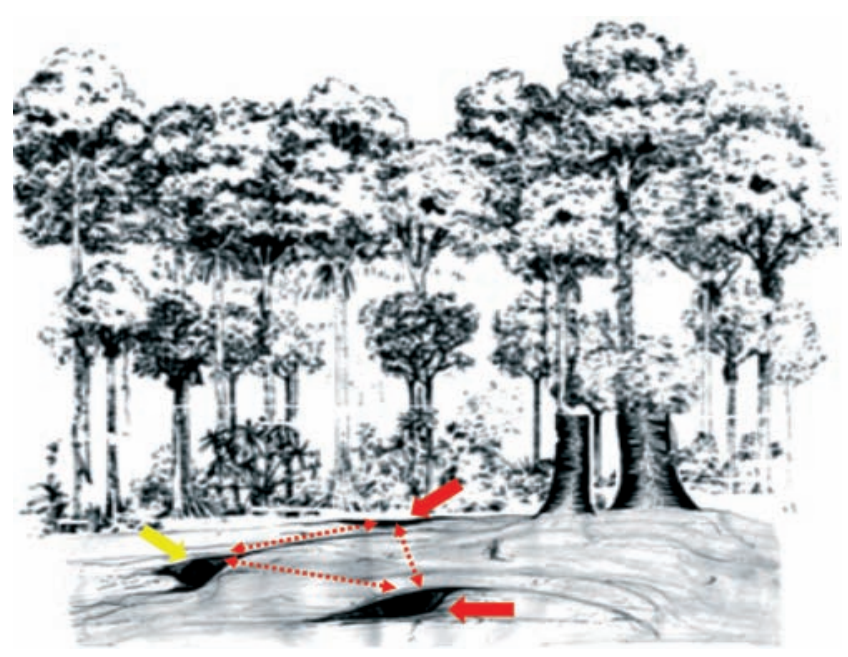

Figura 4. Gráfico de un ambiente de dormir de la forma Ac. Las flechas amarilla y roja indican el orificio de uso habitual y de fuga, respectivamente y las líneas roja punteadas la dirección de los túneles que conectan con los orificios. (Dibujo de Meléndez G, 2006-2007)

a través de túneles con los orificios de uso habitual y de fuga, respectivamente (Fig. 4). La particularidad de esta forma radica en su origen, ya que fue producto de la erosión del suelo por las precipitaciones fluviales, por lo que el orificio de uso habitual se encontraba en posición apical, mientras que el dormidero estaba ubicado a un costado de la cavidad interna, lo que evitaba su anegación por la escorrentía durante las precipitaciones fluviales. Esta forma mayormente registrada en bosque de colina baja con frecuencia fue observada muy cerca a los cuerpos de agua, por lo que los orificios de fuga generalmente estaban direccionados hacia el curso de las aguas.

Forma Ad: registrada en montículos de tierra entremezclada con troncos y raíces de árboles de gran porte, dando aspecto de repisas como consecuencia de la descomposición avanzada del duramen de los troncos (Fig. 5). Una de las peculiaridades de esta forma fue la cavidad interna que se encontraba en el montículo de tierra, mientras que el tronco fue utilizado para la fuga y en otros casos para el acceso y salida de la cavidad interna. Los orificios de uso habitual fluctuaron de uno a tres y siempre estuvieron libres de hojarascas, en tanto que los dos a más orificios de fuga podían estar o no conectados a la base del fuste. Por otro lado, la longitud de los túneles que conectaban con los orificios de fuga variaron entre 0,60 a 1,0 $\mathrm{m}$, lo que indica su proximidad al dormidero.

\section{Tipo B}

Conformado por huecos en montículos de tierra entremezclado con raíces de árboles de gran porte y expuesta por caída natural. Esta combinación de sustratos (tierra, raíces y hojarascas) proporcionaba protección a la cavidad interna, particularmente en la parte superior del ambiente de dormir que funcionaba a manera de "techo", evitando la escorrentía y brindando condición óptima para el resguardo ante sus depredadores. Los orificios de uso habitual estaban generalmente descubiertos y los de fuga circunstancial tapado con hojarascas. En este tipo de ambiente de dormir, el acceso hacia la cavidad interna lo hacía hasta por tres orificios y para la fuga circunstancial contaba con dos y excepcionalmente tres orificios. Para este tipo se han diferenciado dos formas:

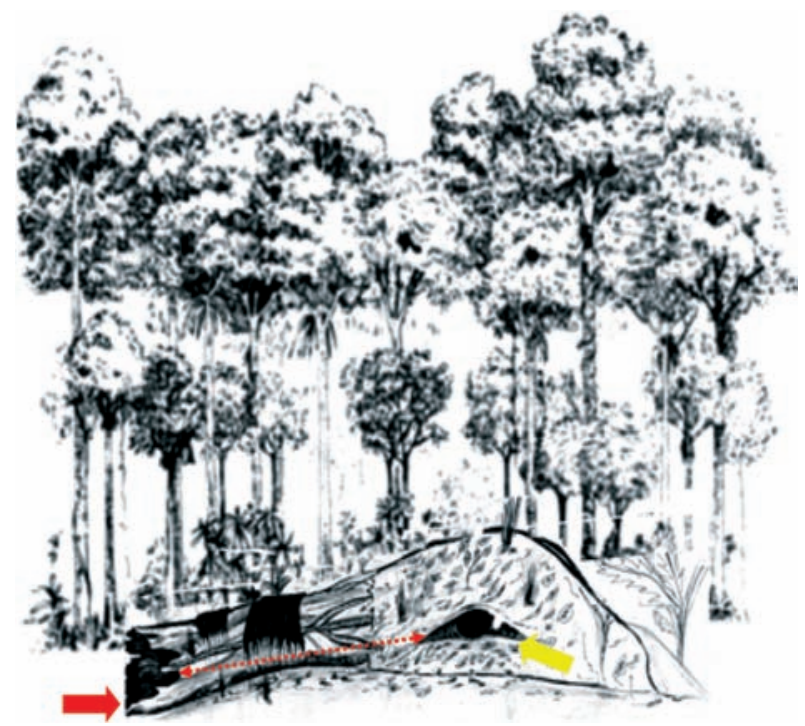

Figura 5. Gráfico de un ambiente de dormir de la forma Ad. Las flechas amarilla y roja indican los orificios de uso habitual y fuga, respectivamente y la línea roja punteada el túnel que conecta ambos orificios. (Dibujo de Meléndez G, 2006-2007)

Forma Ba: en esta forma el orificio de uso habitual y de fuga circunstancial no estaban conectados por túneles, de modo que la cavidad interna donde se encontraba el dormidero estaba conectada directamente con los orificios(Fig. 6). El ingreso y salida de rutina lo hacía a través de un solo orificio, pero para la fuga contaba hasta con dos orificios. Por otro lado, la capa superior o techo estaba compuesta por un enmarañado de raíces muy finas y diminutas y sobre ellas había una capa delgada de hojarascas entremezclada con tierra lo que impedía la escorrentía hacia la cavidad interna, pero también favorecía para una rápida huida en caso necesario. Esta forma de ambiente para dormir con frecuencia se observaron en bosques de terraza alta y de colina baja.

Forma Bb: con aspecto de repisa, donde el manojo de raíces de los árboles proporcionaba espacios vacíos dando origen a la cavidad interna. Una de las características de este ambiente

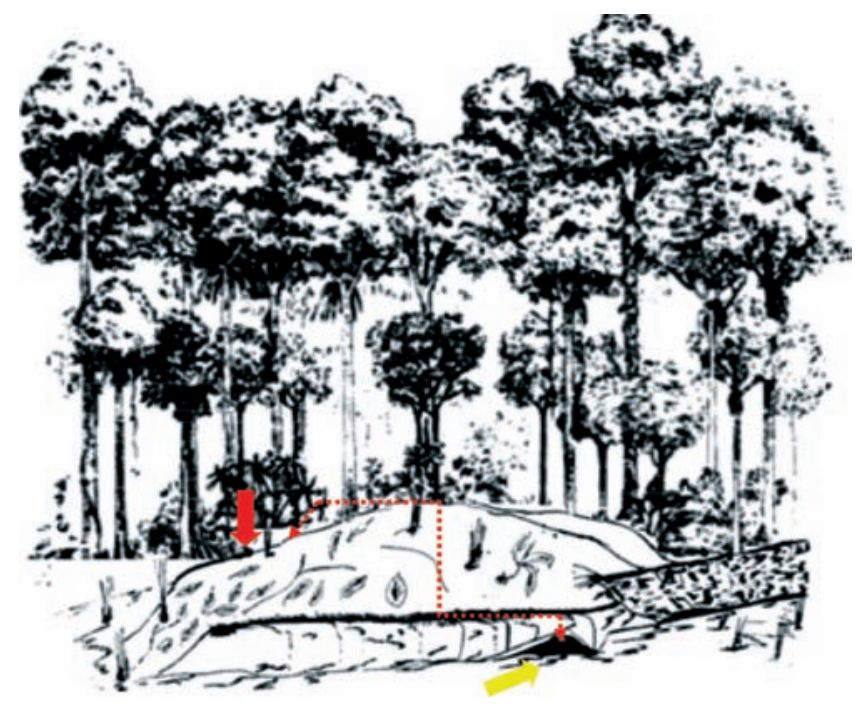

Figura 6. Gráfico de un ambiente de dormir de la forma Ba. Las flechas amarilla y roja indican el orificio de uso habitual y de fuga, respectivamente y las líneas punteadas roja la cavidad interna que estaba conectada con los orificios. (Dibujo de Meléndez G, 2006-2007) 


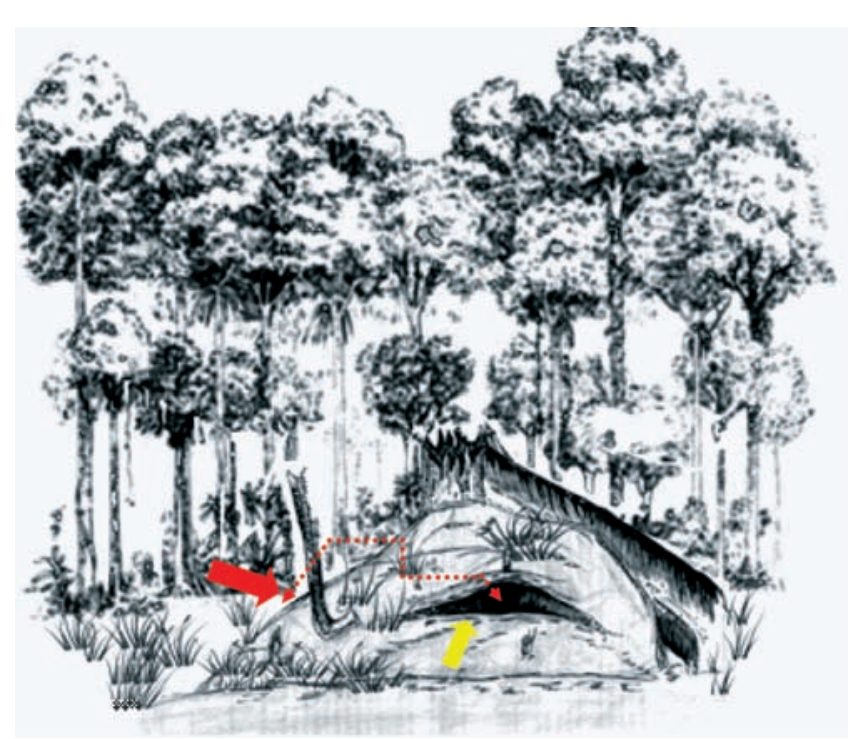

Figura 7. Gráfico de un ambiente de dormir de la forma $\mathrm{Bb}$. Las flechas amarilla y roja indican el orificio de uso habitual y de fuga, respectivamente y la línea roja punteada la conexión de la cavidad interna con los orificios. (Dibujo de Meléndez G, 2006-2007)

fue la ausencia de túneles, por lo que en la mayoría de los casos los orificios de fuga se encontraban en la parte media o cerca del techo de la cavidad interna (Fig. 7). El orificio de entrada hacia la cavidad interna se caracterizó por su forma semicircular y de mayor diámetro que los orificios de fuga que varió de uno a tres. Por otro lado, la capa superior que protegía la cavidad interna era de textura arcillo arenosa y soportada por un enmarańado de raíces gruesas lo que impedía la escorrentía. En algunos casos la base del tronco interiormente vacío formaba paredes del ambiente de dormir dando protección al animal, en tanto que el orificio de entrada y salida generalmente estaba ubicado en las paredes laterales del tronco.

\section{Tipo C}

Conformado por huecos por debajo de raíces de árboles en pie, expandidas horizontal y superficialmente. Este tipo de ambientes tenían desde uno a tres orificios para el uso habitual, todos libre de obstáculos y desde uno a cuatro orificios para la fuga circunstancial, siempre tapados con hojarascas. Para este tipo de ambientes fueron diferenciadas dos formas:

Forma Ca: ubicadas debajo de raíces de árboles frondosos y de palmeras de pona (Iriartea sp.), cashapona (I. sapium), remocaspi (Swartzia brachyrachis), entre otros (Fig. 8). En esta forma había un orificio para el acceso hacia la cavidad interna y desde uno hasta cuatro para la fuga circunstancial. Ambientes de esta forma generalmente fueron hallados en bosque de galería, de modo que los orificios de fuga estaban direccionados hacia los cuerpos de agua. Por otro lado, en la cavidad interna había un pequeño espacio compuesto por un colchón de hojarascas que era utilizado como dormidero. La ubicación de estos ambientes de dormir al parecer tenía relación con el dosel de los árboles que era muy cerrado, proporcionando así una magnífica cobertura para la lluvia, evitando la escorrentía hacia la cavidad interna.

Forma Cb: con aspecto de pequeña montańa,formada por el manojo de raíces gruesas de árboles en pie y una delgada capa

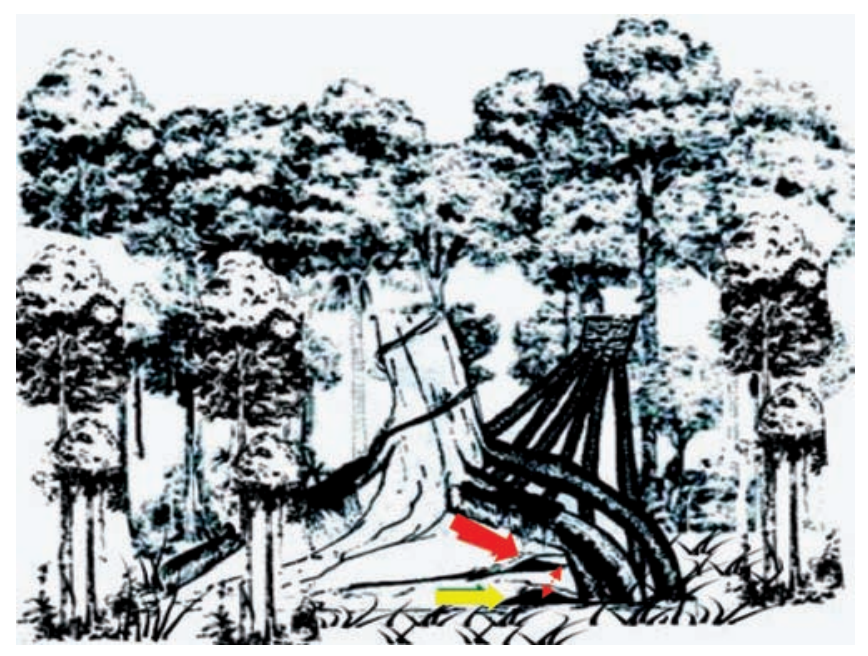

Figura 8. Gráfico de un ambiente de dormir de la forma Ca. Las flechas amarilla y roja indican el orificio de uso habitual y de fuga, respectivamente, los mismos que están conectados directamente con la cavidad interna. (Dibujo de Meléndez G, 2006-2007)

de tierra de textura arcillo - arenoso; generalmente con un orificio para el uso habitual libre de obstáculos y de dos a tres orificios para la fuga circunstancial, todos tapados con hojarascas y ubicados preferentemente en la parte opuesta de las sobresalientes raíces y en algunos casos por detrás de la base del fuste (Fig. 9). En esta forma, los orificios para la fuga circunstancial en la mayoría de los casos estaban directamente conectados con la cavidad interna por ausencia de túneles.

\section{Tipo D}

Conformado por huecos en troncos de árboles y palmeras caídos y en proceso de descomposición, cuyos orificios para el uso habitual y fuga circunstancial en algunos casos se encontraban libres de objetos y en otros cubiertos parcial o totalmente por hojas menudas. Para este tipo se diferenció dos formas particulares:

Forma Da: corresponde a los huecos en troncos de árboles de gran porte, donde los orificios de uso habitual se caracte-

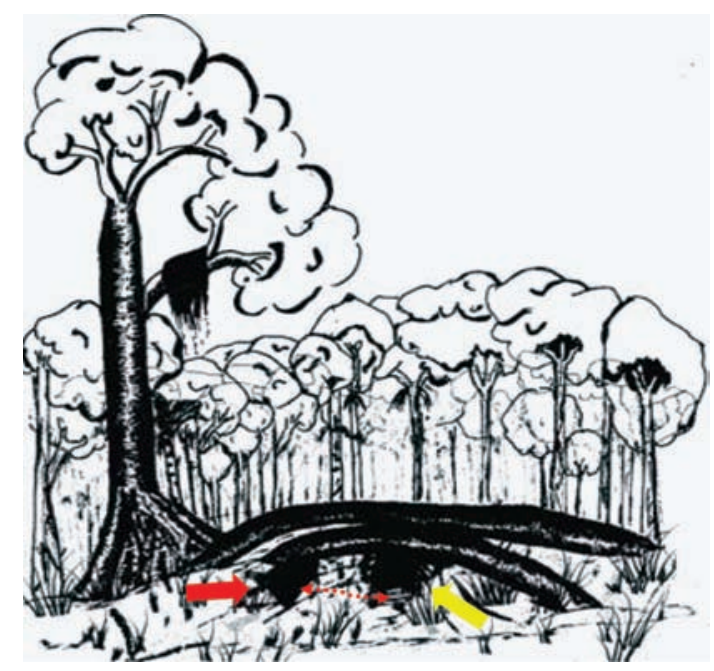

Figura 9. Gráfico de un ambiente de dormir de la forma $\mathrm{Cb}$. Las flechas amarilla y roja indican los orificios de uso habitual y de fuga, respectivamente y la línea roja punteada la conexión de la cavidad interna con ambos orificios. (Dibujo de Meléndez G, 2006-2007) 


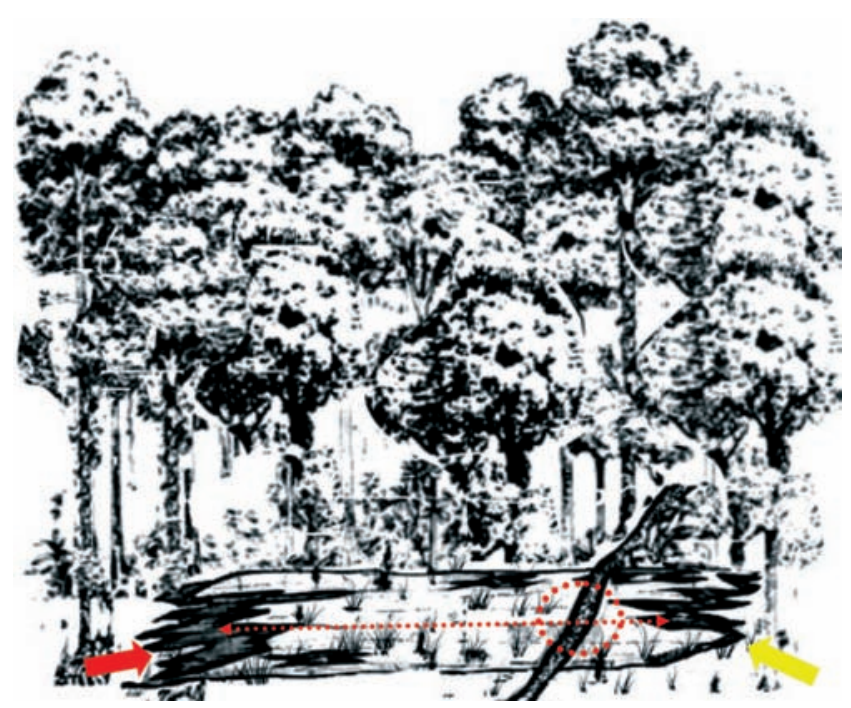

Figura 10. Gráfico de un ambiente de dormir de la forma Da. Las flechas amarilla y roja indican el orificio de uso habitual y de fuga, respectivamente, la línea roja punteada la cavidad interna y el círculo punteado el dormidero. (Dibujo de Meléndez G, 2006-2007)

rizaron por ser unidireccional (Fig. 10). En esta forma la cavidad interna generalmente no presentaba modificaciones y en el sitio utilizado como dormidero siempre se encontró un colchón de hojarascas. La cavidad interna algunas veces estaba húmeda y en otras había hasta pequeños charcos de agua, mientras que los orificios para la fuga circunstancial se encontraban en las paredes laterales del tronco. En esta forma de ambiente de dormir fue la única vez que se halló a este roedor cohabitando con un anfibio relativamente grande conocido con el nombre vernáculo de hualo (Lepthodactylus sp.). Esta forma fue observada con frecuencia en los bosques de terraza alta y en bosque de galería.

Forma Db: corresponde a los huecos de troncos caídos de palmeras de huicungo (A. huicungo), aguaje ( $M$. flexuosa), pona (Iriartea sp.) y ungurahui (J. bataua). En esta forma, el orificio que se encontraba en uno de los extremos era para el uso habitual de modo que se encontraba libre de hojarascas, mientras que el orificio del otro extremo era para la fuga circunstancial, por lo que estaba tapado con hojarascas (Fig. 11). Esta forma de ambiente de dormir fue poco común y mayormente se observaron en bosque de terraza alta con predominancia de palmeras.

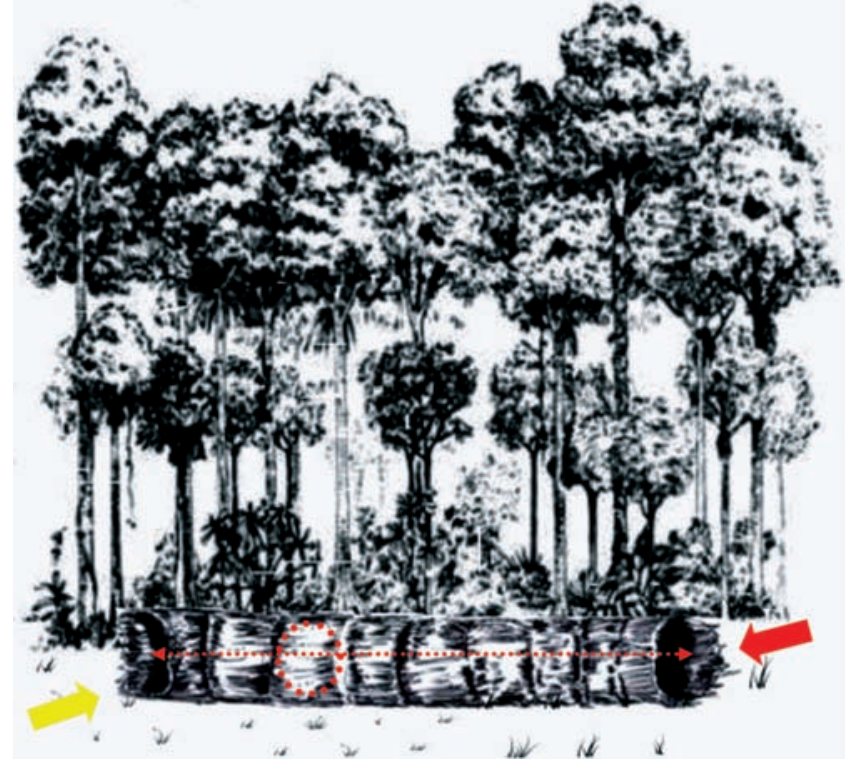

Figura 11. Gráfico de un ambiente de dormir de la forma Db. Las flechas amarilla y roja indican los orificios de uso habitual y de fuga respectivamente, la línea roja punteada la cavidad interna y el círculo rojo el dormidero. (Dibujo de Meléndez G, 2006-2007)

Frecuencia de los tipos de ambientes de dormir.- De los cuatro principales tipos de ambientes de dormir diferenciados y utilizados por el majás ( $C$. paca), los tipos A y C fueron los más frecuentes con 35 y 15 registros equivalentes al 47,2\% y $20,3 \%$, respectivamente; el resto estuvieron presentes pero en menor proporción (Tabla 1). Los resultados también indican a los ambientes de dormir con un solo orificio para el uso habitual y con dos para la fuga circunstancial como los más comunes en el área de estudio (Tabla 1).

Niveles de concentración por tipos de hábitats.- Los registros de ambientes para dormir en los diferentes tipos de hábitats y las observaciones adicionales permiten sostener que la mayor o menor concentración estuvo en estrecha relación con el tipo de hábitat y la producción de frutos, por lo que se ha considerado conveniente establecer niveles de concentración en función de los factores antes mencionados, la misma que fue guiada por las descripciones establecidas por Encarnación (1993). En tal sentido, para el área de estudio fueron diferenciados hasta tres niveles, desde la más alta (nivel I) hasta la más baja concentración (nivel III).

Tabla 1. Frecuencia de los tipos de ambientes de dormir observados en la cuenca del río Alto Itaya.
Usos
\# de orificios
\# de registro por tipo de ambiente de dormir
Total
$\%$

\begin{tabular}{|c|c|c|c|c|c|c|c|}
\hline & & $\mathrm{A}$ & $\mathrm{B}$ & $\mathrm{C}$ & $\mathrm{D}$ & & \\
\hline \multirow{4}{*}{ Habitual } & 1 & 29 & 11 & 12 & 11 & 63 & 85,2 \\
\hline & 2 & 4 & 0 & 1 & 1 & 6 & 8,1 \\
\hline & 3 & 1 & 1 & 2 & 0 & 4 & 5,4 \\
\hline & 4 & 1 & 0 & 0 & 0 & 1 & 1,3 \\
\hline \multicolumn{2}{|c|}{ Total } & 35 & 12 & 15 & 12 & 74 & 100 \\
\hline \multirow{4}{*}{ Circunstancial } & 1 & 18 & 4 & 3 & 9 & 34 & 46 \\
\hline & 2 & 13 & 6 & 5 & 2 & 26 & 35,1 \\
\hline & 3 & 3 & 2 & 6 & 1 & 12 & 16,2 \\
\hline & 4 & 1 & 0 & 1 & 0 & 2 & 2,7 \\
\hline \multicolumn{2}{|c|}{ Total } & 35 & 12 & 15 & 12 & 74 & 100 \\
\hline
\end{tabular}


Nivel I: correspondió a los bosques adyacentes y contiguos al río y quebradas con inundación efímera y periódica estacional e incluye el denominado bosque de galería con piso más o menos compacto. En este tipo de bosque caracterizado por su alta producción de frutos, en particular de palmeras como huasaí (Euterpe sp.), pona (Iriartea sp.) y huicungo (A. huicungo), fueron registrados 41 ambientes de dormir equivalente al 55,4\%, convirtiéndose así en el óptimo hábitat para este roedor.

Nivel II: correspondió al bosque de terraza alta con grandes extensiones de terrenos planos u ondulados con disecciones leves no inundables ni expuestas al flujo periódico y estacional de las masas de agua. En estos bosques caracterizado por su relativa abundancia de frutos, en particular azúcar huayo (Hymenaea sp.), leche huayo (Couma macrocarpa), yahuar huayo (Rhigospira sp.), naranjo podrido (Parahancornia sp.) y machimango (Eschweilera spp.), fueron registrados 25 ambientes de dormir equivalente al $33,8 \%$, por lo que constituye el segundo hábitat más importante para este roedor.

Nivel III: correspondió al bosque de altura más extenso denominado bosque de colina baja, cuya vegetación de tipo varillal estuvo conformado por árboles de fuste delgado y recto y cubierto con abundantes plantas epífitas y por enmarañados de bejucos y lianas; piso cubierto por un colchón de hojarascas y sotobosque más o menos cerrado y poblado por irapay ( $L e-$ pidocaryum tenue). En este tipo de bosque caracterizado por su baja producción de frutos fueron registrados 8 ambientes de dormir equivalente al 10,8\%, lo que demuestra que se trata de un hábitat poco apropiado para este roedor.

\section{Discusión}

De los diferentes tipos de ambientes de dormir diferenciados para el majás (C. paca), el tipo A fue el de mayor registro, quizá por la disponibilidad de huecos pre construidos, resultado que coincide parcialmente con los registros de Pérez (1992) y Moreira y Macdonald (1997). Sobre el particular, Yockteng (1982), Emmons (1990), Voss y Emmons (1996), Aquino et al. (2001) y Oliveira y Bonvicino (2006), sostienen que el ambiente de dormir de este roedor esta conformado por huecos que se encuentran preferentemente en montículos de suelos arcillosos, pero también en troncos de árboles caídos, entrecruzamiento de raíces de árboles y en depresiones del suelo. Por otro lado, Emmons (1990)y Moreira y Macdonald (1997) sostienen que el ambiente de dormir de este roedor cuenta con un orificio principal y con varios orificios secundarios ocultos con hojas secas, apreciación que coincide en términos generales con los resultados obtenidos en el presente estudio, pero contrasta a nivel de especificidad, debido a que los autores antes mencionados no detallan el número de orificios para el uso habitual y para la fuga circunstancial, tampoco hacen mención de la conexión de estos orificios con la cavidad interna donde se encontraba el dormidero. Estos resultados tienen mayor coincidencia con las sostenidas por Aquino et al. (2009), Leopold (1977), Emmons (1990) y parcialmente con Eisenberg (1989). Sin embargo, con excepción de los dos primeros, el resto tampoco hace mención que los orificios escondidos y tapados con hojas son usados para la fuga circunstancial ante el peligro inminente de ser presa de sus depredadores. En referencia a ciertos hábitos, Alvares del Toro (1952) y Yockteng (1982) mencionan que este roedor no come en el sitio donde recoge el alimento, sino que lo traslada a otro lugar escogido de antemano donde instala su comedero, pero ni ellos ni otros autores hacen referencia de que este roedor tampoco tiene por costumbre defecar, orinar y menos comer al interior de su ambiente de dormir como se demuestra en este estudio.

En la cuenca alta del río Itaya los ambientes de dormir del majás fueron relativamente abundantes, lo que confirma su alta densidad poblacional (Aquino et al. 2009), la misma que tendría relación con ciertos factores, entre ellas la existencia de un sin número de quebradas, cuyas aguas sirven como medio de defensa cuando son acosados por sus depredadores; escasa presencia de depredadores naturales debido a la alta presión de caza de mamíferos de tamańo grande (Aquino et al. 2007) y presencia de bosques de inundación temporal, hábitat preferido por este roedor por la alta producción de frutos. Sobre el particular, Voss y Emmons (1996), hacen mención que la variación en las características de la vegetación puede ocasionar cambios en los patrones de uso del hábitat; así, la mayor densidad de la vegetación y mayor número de individuos en los estratos de vegetación menores a $7 \mathrm{~m}$ de altura puede traducirse en una mayor capacidad del hábitat en ofrecer mejores condiciones para la construcción de ambientes para el sueño. En nuestro caso, esto explicaría la mayor frecuencia de madrigueras encontradas en el bosque de galería. Estos resultados coinciden con las apreciaciones hechas por Voss y Emmons (1996), quienes sostienen que este roedor puede ser observado en cualquier tipo de bosque, pero con mayor frecuencia en las cercanías de las orillas y en bosques inundables con sotobosque denso. Sin embargo, los resultados también indican la concentración de este roedor en bosques de terraza alta, coincidente con el período de fructificación en tanto que las zonas aledañas a las orillas se encontraban inundadas.

\section{Agradecimientos}

Nuestro agradecimiento al Centro Amazónico de Educación Ambiental e Investigación (ACEER) y al Consejo Superior de Investigación (CSI) de la Universidad Nacional Mayor de San Marcos (UNMSM) por el financiamiento. Nuestro reconocimiento a Gilmer Montero, guía y asistente de campo con quien compartimos buenos y malos momentos durante las actividades de campo.

\section{Literatura citada}

Álvarez Del Toro M. 1952. Los animales silvestres de Chiapas. Ediciones del gobierno del Estado Tuxtlan Gutiérrez, Chiapas.

Aquino R. \& F. Encarnación. 1986. Characteristics and Use of Sleeping Sites in Aotus (Cebidae: Primates) in the Amazon Lowlands of Peru. Am. J. Primatol., Vol. 11 (4): 319-331.

Aquino R., C. Terrones, R. Navarro \& W. Terrones. 2007. Evaluación del Impacto de la caza en mamíferos de la cuenca del río Itaya, Amazonía Peruana. Rev. Peru. Biol. 14(2): $181-186$.

Aquino R., D. Gil \& E. Pezo. 2009. Aspectos ecológicos y sostenibilidad de la caza del majás (Cuniculus paca) en la cuenca del río Itaya, Amazonía peruana. Rev. Peru. Biol.16(1): 67- 72.

Aquino R., R. E. Bodmer \& G. Gil. 2001. Mamíferos de la Cuenca del Río Samiria: Ecología Poblacional y Sustentabilidad de la Caza. Imprenta Rosegraf, Lima, Perú.

Bendayán N. \& J. Bardales. 2004. Impacto del uso de carne de monte en el área de Influencia a las localidades de Iquitos, Nauta y Tamshiyacu, Loreto - Perú. Tesis para el Grado de Magister en Ciencias, Universidad Nacional de la Amazonía Peruana. 
Bendayán N. 1991. Influencia socioeconómica de la fauna silvestre en Iquitos, Loreto. Tesis para el título de Biólogo. Universidad Nacional de la Amazonía Peruana

Bodmer R. E., P. Puertas, C. Reyes, J. García \& D. Días. 1997. Animales de caza y palmeras integrando la socioeconomía de extracción de frutos de palmeras y carne de monte con el uso sostenible. En: T. Fang, R. Bodmer, R. Aquino y M. Valqui, eds. Manejo de Fauna Silvestre en la Amazonía. La Paz, Bolivia. Pp. 75 - 86.

Bóer S. 1981. Agutí paca. Las “lapas”. Roedores de America tropical. Natura (70 - 71): 40 - 41.

Eisenberg J. F. 1989. Mammals of the Neotropics: The Northern Neotropics. Vol. 1. The University of Chicago Press.

Emmons L. H. 1990. Neotropical Rainforest Mammals: A Field Guide. The University of Chicago Press, Chicago.

Encarnación F. 1993. El bosque y las formaciones vegetales en la llanura Amazónica del Perú. Alma Mater 6: 95 - 114.

Leopold A. 1977. Fauna silvestre de México. 1ra. Edición. Instituto Mexicano de Recursos Naturales Renovables. México.

Mondolfi E. 1972. Mamíferos de caza de Venezuela. La lapa o paca. Defensa de la naturaleza 2(5):4 - 16.

Moreira R. J. \& D. Macdonald. 1997. Técnicas de manejo de capivaras e outros grandes roedores na Amazônia. En: C. Valladares, R. Bodmer y L. Cullen, eds. Manejo e Conservaçâo da Vida Silvestre No Brasil. Brasil. Pp. 2 - 15.
Navarro R. \& C. Terrones. 2006. Evaluación del Impacto de la caza en mamíferos de la cuenca alta del río Itaya. Loreto - Perú. Tesis para el título de Biólogo,Universidad Nacional de la Amazonía Peruana.

Oliveira J. \& C. Bonvicino. 2006. Orden Rodentia. En: N. Reis, A. Peracchi, P. Wagner \& P. Isaac, eds. Mamíferos do Brasil. Londrina, Brasil. Pp. 382 - 384.

Pérez E. M. 1992. Agouti paca. Mammalia Species 404: 1 - 7.

Puertas P. 1987. Algunos aspectos ecológicos de Aotus vociferans "buriburi" (Cebidae: Primates). Tesis para el título de Biólogo, Universidad Nacional de la Amazonía Peruana.

Terrones W. 2006. Ecología Poblacional del “tocón negro” Callicebus torquatus lucifer (Primates: Phiteciidae) en la cuenca alta del río Itaya, Loreto, Perú. Tesis para el título de Biólogo, Universidad Nacional de la Amazonia Peruana.

Voss R \& L. Emmons. 1996. Mammalian Diversity in Neotropical Lowland Rainforests: A preliminary Assessment. Bull. Amer. Museum Nat. Hist. No. 230.

Yockteng C. 1982. Observaciones ecológicas y ensayos en zoo criadero del "majás" (Agouti sp.). En: S. Moller-Hergt,ed. Investigación y utilización de la fauna silvestre del bosque húmedo tropical. Libro III, 1ra. Edición. Comité Nacional de Programa el Hombre y La Biosfera: Proyecto UNESCO-PNUDA. Loreto, Perú. Pp. 121-154. 